\title{
Killing animals as a necessary evil? The case of animal research
}

\author{
Nuno H Franco, I Anna S Olsson,
}

Originally published in The end of animal life: a start for ethical debate. F.L.B. Meijboom and E.N. Stassen, Editors. 2016, Wageningen Academic Publishers: Wageningen.

\section{ABSTRACT}

This chapter addresses the question of killing animals in research, primarily from a moral perspective, but also taking into account some of the practical and scientific considerations with moral consequences in this context. We start by exploring in which situations animals are killed in research and whether these are always inevitable, analysing re-use and re-homing of animals as potential alternatives. We then discuss for whom - and under what circumstances -killing matters, considering situations where there may be a conflict between the wish to avoid killing and that to avoid suffering, and further take humananimal interactions into account. We argue that, although there are relevant practical, scientific and ethical arguments favouring the euthanasia of animals in most research contexts, there is a potential for rehabilitating more animals than is currently the practice.

\section{INTRODUCTION}

The use of animals in the life sciences seems to be accepted by most people provided that it allows advancing biomedical knowledge, that such advances cannot be achieved using non-animal methods and that animal suffering is kept to a minimum [1, 2]. Nevertheless, animal experimentation remains a controversial issue. While the use of animals in general and the welfare of these animals have been subject of wide debate, within the discussion of animal experimentation the moral implications of killing animals, have not been given as much attention.

Typically, animals used in research are euthanized at the end of the experiments. The most recent statistics [3] regarding the scientific use of animals in the European
Union point to a total of 11.5 million vertebrates used in 2011 in all fields of basic and applied biomedical science, as well as in education and training, in both the public and the private sector. Considering the size of EU27 population - 502.5 million people in 2011 [4] - this gives a ratio of roughly 2,3 vertebrates (mostly rodents and fish) used per 100 EU citizens every year. This makes the annual number of animals used in Europe for all scientific and educational purposes but a very small fraction of that of those - mostly cows, pig, sheep, poultry and fish - killed for food in the EU daily [for statistics see $5,6,7]$. Of course, the fact that the overwhelming majority of people (including those concerned about animal welfare) seem to approve of the killing of animals for food, is not a reason to dismiss ethical concerns over the killing of animals in research. First, the use of animals may be more readily and easily replaced in food production than in biomedical research [8], at least as regards the nutritional value. Maybe more important, using the majority view as moral guidance is questionable to say the least. Also, whereas meat production without killing seems inconceivable, research and experiments ${ }^{1}$ may not necessarily require the curtailing of animals' lives. Furthermore, the fact that the number of animals killed in experiments pales in comparison to the vast numbers killed in common human activities outside the laboratories does not remove our moral responsibilities towards these animals.

\footnotetext{
1 This chapter refers specifically to the killing of animals in a biomedical context. Although equally important, other contexts where animal research takes place, such as research on farm animals, present distinct ethical, technical and social implications which we do not address here.
} 


\section{IB: IBMC}

Starting from the assumption that at least some animal research is relevant, ethically acceptable and presently not replaceable, some harm to animals in research may be perceived as a 'necessary evil', in particular in face of the moral importance of advancing biomedical knowledge for the benefit of humans and non-humans alike. However, it should nevertheless be reflected upon in which circumstances it may - or may not - be either 'necessary' or 'evil' to kill animals in the context of animal research. In this chapter, we discuss whether killing is inevitable, or morally problematic, as well as to whom this killing matters.

\section{Killing animals in research - is it always inevitable?}

In order to answer the question of whether killing is inevitable, it becomes necessary to understand in which situations, and for what reasons, animals are killed in research. The majority of cases fall into three main categories, namely a) when the research as such requires that animals are killed, b) when killing is (or is considered to be) in the animals' best interest to prevent further suffering, and c) when killing results from a contingency - of financial, logistic, technical or even cultural nature - secondary to the scientific process, per se. The first two cases present a scientifically and ethically more convincing argument for the euthanasia of animals, as the unavoidability of killing in these cases is taken as a starting premise. However, alternative approaches to some experimental procedures that typically require killing may be considered.

The killing of laboratory animals is often elicited by the need to collect appropriately sized biological samples in smaller species, like rodents or fish, where for example the entire volume of blood may be required for analysis. When this is combined with the need to collect samples at different time points, an animal - or a group of animals - for each time point must be killed. In some cases, necropsy is needed to assess the internal alterations caused by disease. When using larger animal species, however, repeated sampling can be accomplished without having to kill different animals at several time points, with the added advantage that the quality of the experimental outcome may be improved by minimizing the effect of inter-individual variability, since the same animal is used as its own control [9, 10]. Necropsy can also sometimes be replaced by use of imaging technologies to follow disease progress in the living animal, while avoiding having to kill animals for this purpose. One example is the use of imaging technologies to follow the progress of developing infections such as in tuberculosis research [11-13] as well as the growth of tumours in both rodents and non-human primates. This allows reducing considerably the number of animals otherwise needed for post-mortem analysis at different time points.

Laboratory animals may also be killed in order to prevent them from unnecessary suffering. A paramount example is the use of 'humane endpoints', generally understood as the euthanasia of research animals when their health and welfare reach a previously defined threshold level of pain or suffering. Humane endpoints are particularly important in studies on progressive diseases, as they prevent that animals reach advanced stages and subsequently die from the disease or associated conditions (e.g. starvation or dehydration due to inability to reach the food hopper; or attack by less affected cage mates). They may also be applied in response to unexpected welfare problems requiring emergent intervention (resulting from injury, procedural errors or sudden aggravation of clinical signs). In such cases, when animals would otherwise suffer and this suffering cannot be avoided in any other way, the early killing of research animals is generally considered to be the best practice, as well as often legally required [14].

Laboratory animals are however sometimes killed for what seems to be rather trivial reasons. One derives from a tendency to use only animals of one sex. This is sometimes females, in order to avoid aggression-related problems with group-housed males $[15,16]$ but more often males are preferred [17, 18]. In any case, preference for a given sex can lead to the culling of animals of the other sex. Also, from a scientific perspective, using both male and female animals in research is valuable since it allows detecting possible sex differences, which with appropriate experimental design can be achieved without using additional animals. Such routine culling of healthy animals may also result from insufficient planning of experiments, since one reason animal facility staff and commercial breeders breed animals in excess is to be able to supply them on demand, on account of researchers' often requesting animals on very short notice [19]. 
In a number of situations, killing laboratory animals is neither necessary to obtain scientific information nor to prevent further suffering. In such cases, reusing laboratory animals for other experiments or research projects can be an alternative to killing them, provided full rehabilitation is possible. Through this approach, fewer animals are killed after each experiment, and fewer naïve animals - which otherwise would also be killed - need to be bred. There may be limited margin to re-use small laboratory animals because of the limitations for sampling and the need to sacrifice them to obtain tissues, and the low costs for breeding new mice also makes the use of naïve animals for experiments a preferred option, Therefore in practice, reuse of large, non-rodent mammals is more common, in particular in toxicology, pharmacokinetic studies or studies in which telemetry is used [19-22].

Reuse has the potential to not only avoid the early termination of animal life, but also reduce the overall number of animals used in research, thus saving time and resources [23, 24]. Also, with appropriate experimental design, reuse of animals can provide statistically powerful studies with a small number of animals [20, 25, 26]. However, reusing animals raises ethical and methodological issues when compared to the use of naïve animals, which have not been subject to previous experiments and must therefore be considered on a caseby-case basis [19, 24, 26]. This includes defining the upper threshold of welfare impact that animals will experience as a result of cumulative experimental use, as contemplated in current EU legislation [27]. This should take into account that while some measures may minimize the cumulative effect of repeated sampling - such as the use of permanent devices (e g permanent catheters and telemetry devices) [25] - overall repeated experiments usually imply greater welfare impact, and hence the harms elicited by the reuse, or continuous use, of the same animals for long periods of time should always be carefully weighed against the harms of successively using naïve animals, for shorter periods [19]. Taken together, these considerations point to a conflict between using (and typically killing) a greater number of animals at a smaller welfare cost to each animal or using (and killing) a smaller number of animals at a greater cost to each, i.e. between Reduction and Refinement (see the next section of this chapter and Franco and Olsson [28] and Franco et al 2014 [29] for further discussion of this conflict).

Another option to avoid the killing of animals after their use in research is to re-home them as companion animals or to sanctuaries [30]. Such re-homing is still far from becoming mainstream practice, with the exception of the retirement of chimpanzees to sanctuaries. In the USA, the main country in which chimpanzees are used in research, the euthanasia of surplus chimpanzees under the care of federal public agencies is forbidden and a fund was established for the setting up and maintenance of a sanctuary system for the retirement of these chimpanzees. The present cultural status quo may make people having difficulties in envisaging purpose-bred laboratory animals outside their scientific role and therefore to consider them appropriate for a life outside the laboratory [31]. However, there are several examples of successful programs for re-homing of laboratory cats and dogs into family homes and non-human primates into sanctuaries [30,32-37], showing that it can be a feasible option.

A few legislative pieces reflect a strong position as regards avoiding killing, one of them being the German 'Animal Welfare Act', the Tierschutzgesetz, which forbids the killing of any vertebrate without a "sound reason". Several reasons are however deemed 'acceptable' for the killing of animals in German legislation, including the slaughter of animals for food, hunting, or research purposes. Also, the rights of citizen consecrated in German law - and which includes the right to do research - take precedence over the Tierschutzgesetz $[38,39]$. This legislation has, however, served as a legal framework in Germany for the rehoming of laboratory animals after their use in scientific research [35]. Rehabilitation of non-human primates, cats, and dogs used in research is also encouraged (although not required) in present EU legislation regulating animal use in science [27].

A bolder approach has been put forward by the 2006 amendment of the Indian law regulating animal experiments. This legislative document makes those using large animals in scientific experiments responsible for what happens to animals not only before and during, but also after experiments [40,41]. Thus, it becomes mandatory for 


\section{IB:IIBMC}

researchers using dogs, cats, sheep, goats, cattle, horses, non-human primates or other large animal species to cater for their aftercare and rehabilitation, allowing them to "resume a normal existence", unless euthanasia is deemed necessary. In India, rehabilitation costs must be included in the research budget through an estimate of the statistically expected life span of the animals and scaled per animal in positive correlation with level of sentience. However, while sentience is considered as a criterion for rehoming conditions, the rehabilitation of (presumably equally sentient) small laboratory mammals is deemed as "not necessary", although it may be open to consideration by institutional animal ethics committees.

After having considered the different situations under which animals are killed, and having argued that these are not always a scientific or animal welfare necessity, we now turn to the moral issue raised by the killing of animals in scientific experiments.

Is it morally 'wrong' to kill animals in research?

In the previous section we focused on practical issues as regards finding alternatives to the early curtailing of animals' lives in research. It remains to be discussed whether, or to which degree, killing an animal is, or may become, a moral issue.

Historically, the Western tradition of thinking does not consider the killing of non-humans morally problematic in itself. The Judaeo-Christian religious moral tradition held that while one must abstain from cruelty, killing animals was not in itself morally problematic, as animals lacked an 'immortal soul' [42]. In the secular anthropocentrism that would follow from the seventeenth century on, cruelty towards animals would continue to be considered morally condemnable but, as Immanuel Kant would state, those "who use living animals for their experiments, certainly act cruelly, although their aim is praiseworthy, and they can justify their cruelty, since animals must be regarded as man's instruments" [43]. Even Jeremy Bentham, founder of utilitarian moral philosophy ${ }^{2}$ would not state animal research to be unethical, provided the experiment had "a determinate object, beneficial to mankind, accompanied

\footnotetext{
${ }^{2}$ In proposing sentience as the primary criterion for defining to whom should be given moral consideration, Bentham built the philosophical framework within which Peter Singer operated in his seminal work AnimalLiberation [44]
}

with a fair prospect of the accomplishment of it", thus acknowledging humans had certain precedence over other animals [45].

The present mainstream approach to animal use in research is predominantly utilitarian in nature, and in general greater attention is given to preventing the suffering of animals than to avoiding their killing. This may sometimes have quite far-reaching consequences. Under current European legislation regulating animal use in experiments, killing laboratory animals is not even - by definition - considered to be a procedure, if no other prior interventions are carried out [27]. This is consistent with the predominant welfarist view of good practice in research with animals, under which the painless killing of laboratory animals poses no ethical problem, or at least not from a welfare point a view, as non-existence inheritably implies absence of negative experiences. Following the welfarist approach, conducting experiments under terminal anaesthesia is seen as an ethically preferable approach, as animals are not aware of any aversive stimuli, and are spared the distress associated with recovery from anaesthesia and other interventions.

However, the assumption that death in itself is not a moral problem is not as clear-cut for every researcher, and for every species. When nineteenth-century physician George Hoggan said he would be "inclined to look upon anaesthetics as the greatest curse to vivisectible animals", he alluded to the fact that anaesthesia allowed researchers to use great numbers of animals without further moral quandaries. More recently, James Yeates challenged the mainstream idea that the swift and painless killing of animals is not a welfare issue, defending instead that there are at least some instances in which killing animals poses a welfare problem. He bases his argument by following the rationale that it is in an animals' interest to not only avoid negative feelings but also experience positive ones, from where it follows that as long as the animal would be expected to have a life worth living, death deprives the animal of the positive feelings it would otherwise experience in its lifetime [46].

Animal research presents a number of situations where there is an apparent conflict between avoiding killing and 


\section{IB:IIBMC}

preventing suffering. While Reduction and Refinement ${ }^{3}$ may often go hand-in-hand, there are several instances in which these principles conflict with each other. If one were to be governed by the "badness of killing" argument, one would as far as possible avoid taking the lives of animals, thus giving precedence to Reduction. However, it is more widely recognized that Refinement should be prioritized if Reduction efforts result in a significant burden for each individual animal, that would otherwise be avoided or minimized by using more animals [28, 48, 49]. In fact, by requiring that the application of Reduction should take "into account individual animal welfare in relation to minimizing pain, suffering, distress or lasting harm", current texts partly address this conflict and establish that avoidance of killing should not come at the expense of animal suffering.

Nevertheless, this precedence is not consensual. Having the Refinement/Reduction conflict in mind, we presented a markedly dichotomic reduction/refinement dilemma to participants in 11 laboratory animal science courses held in Portugal between 2008 and 2011 ( $\mathrm{N}=235)$. Asked whether they would rather use twenty mice on a painful experiment (but with no permanent physical damage) or use the same mouse in repeated measures (assuming this would not impact the validity of the study), $49 \%$ would rather use the same mouse, while $51 \%$ preferred to divide the burden by 20 mice. A proportion of researchers would however reconsider and reuse the same animal if it were a dog $(31 \%)$ or a non-human primate $(38 \%)$. But even if the proportions are different for different species, the overall picture is still that these animal researchers divide into a group who give preference to avoiding suffering and another group preferring to avoid killing. ${ }^{4}$

\footnotetext{
${ }^{3}$ Replacement (of animal experiments with alternative approaches), reduction (of animal numbers) and refinement (of experimental procedures to reduce animal pain, distress and suffering) constitute the 3Rs. First presented in 1959 (Russell and Burch, 1959), this is now a widely accepted governing principle for animal research.
}

4 A more comprehensive view of scientists' attitudes to animal research can be found in Franco and Olsson [28], which reports a study conducted on a large part of this sample. For further discussion on the conflict between longevity, value and quality of life in animal research and other contexts, see Franco et al [29]
To whom does death matter?

It seems obvious that the main stakeholders in this issue are the animals themselves; after all it is their lives that are ended or allowed to continue. However, trying to approach the question of killing from an animal-centred perspective means confronting a number of philosophical questions that, for us humans, are troublesome to answer (see [50]). Understanding that one day one's life will end and one will cease to exist is part of the human experience and development. In some way, death seems potentially more harmful to an individual who has this awareness, because they can be harmed also by worrying about the timing, conditions and consequences of death in the future [51]. Our very limited understanding of what death means to an animal is a strong limitation when discussing the ethics of killing. Equally important and difficult to answer is the question of what is a life worth living and when death is preferable to going on living. These questions are developed in more detail in other chapters in this book and we will not attempt to answer them here. However, as the next example will illustrate, even taking a more anthropocentric perspective on animal killing does not completely free us from questions about what death means to animals.

Philosophers such as Bernard Rollin [52] have suggested that humans have established social contracts with domesticated animal species, under which both species have benefited across time, such as in traditional farming [53]. This concept may also be applied to the collaborative relationship between researchers and laboratory animals [54]. It is reasonable to say that, for instance, rodents used in non-invasive experiments and under good husbandry are likely to be, at least in some aspects, better off than their wild counterparts ${ }^{5}$, as they are protected from threats to their wellbeing such as weather conditions, hypothermia, dehydration, starvation, natural predators or pest control and have their basic needs catered for by humans. In the usual interpretation of this hypothetical social contract, humans get to decide when and how to kill animals. If accepting the argument that it is better to have lived a short,

${ }^{5}$ For instance, even if provided with shelter and ad libitum food and water in a semi-natural enclosure [55] the average life span of wild rats (Rattus norvegicus) is about half of that of laboratory rats of the same species [56]. 


\section{IB: IBMC}

but 'happy' life than not to have lived at all, when should we consider that animals do not live a 'life worth living' and we hence fail to our part of the contract? Is the use of animals lives less justified in these cases? If so, and if rehabilitation is possible, does allowing an animal to retire after its scientific purpose help fulfil our duty of fairness to the animals?

Important as they are, the animals are not the only stakeholders in this discussion. The use of animals in research typically requires instrumentalizing them to some degree, but animals are certainly not - and are not perceived as - inert instruments of inquiry. Instead, they are living beings that can establish significant relationships with humans. This animal-human bond is particularly strong between animals and caregivers who, rather than associating animals with a given procedure or test, see these sporadic interventions as only a small fraction of the animals' lives and of their time spent with them. This bidirectional interaction leads staff members to develop an appreciation for the value of the animals' lives, which in turn makes their death matter morally to them [54, 57-60]. Typically, laboratory animal caretakers are also often those who have to kill the animals trusted to their care. The contradiction between these tasks can elicit what A. Arluke has coined as the "caring-killing paradox" [61], characterized by a sentiment of grief, guilt and moral stress, even in situations when euthanasia is performed to prevent debilitated animals of further suffering. Nevertheless, getting to know the animals and establish a bond with them may be important for allowing caretakers to cope with stress and improve animal health, well-being, and the quality of research, in spite of the emotional cost borne by the animal facility personnel.

People working in animal facilities feel particularly downhearted about killing healthy animals for what they may perceive as convenience, as when an experiment is coming to an end, animals are being excluded from an experiment (e.g. for failing to perform a designated task in a behavioural study); or during routine culling of surplus animals in animal facilities, the latter rendering killing a mere management technique. In these situations, and especially when animals have not served any scientific purpose, aside the grief that may arise, killing will also be perceived as 'wrong' to students, veterinarians and caregivers, in particular when a viable adoptive home, or other alternative, is available $[33,59,62]{ }^{6}$

From the human perspective, it seems as if all animals are not equal when it comes to killing. This is evident in the most recent EU legislation regulating animal use, in which it says that "animals such as dogs and cats should be allowed to be rehomed in families as there is a high level of public concern as to the fate of such animals" (Recital 26 [27]). A similar species preference is also shared by animal facility personnel [59, 60] and was also evident among researchers participating in laboratory animal science training. Indeed, many were open to allow several animal species - in particular non-human primates and companion animal species - to be given for adoption or taken to a sanctuary if rehabilitation were possible (Figure 1).

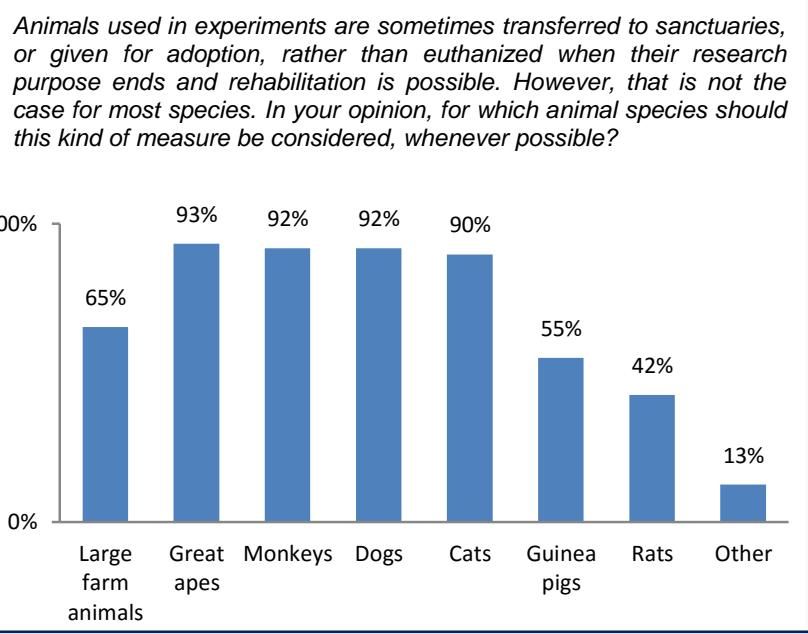

Figure 1. Data from a survey conducted with participants in 11 laboratory animal science courses held in Portugal between 2009 and 2011 ( $n=193$, courses held in four different universities and following FELASA guidelines for either category B or C).

\section{Discussion and conclusion}

In this chapter, we have discussed whether the killing of laboratory animals is always inevitable, whether it is morally problematic and to whom such killing matters. As we demonstrated in the first section, it is often unavoidable to end the lives of research animals, either because the research itself requires this or because the animals would

${ }^{6}$ Researchers, on the other hand, have been described as having more of an ambivalent view $[62,63]$. 


\section{IB: IBMC}

otherwise suffer unacceptable pain and distress. However, animals are sometimes killed when they could have been rehabilitated or even when they are healthy and have not been used in research. In these situations, re-homing into sanctuaries or family homes is a viable option, which is presently not extensively used. In the second section, we discussed the moral implications of killing animals. Even though in the mainstream view on laboratory animal ethics preventing suffering seems to be more important than avoiding killing, killing nevertheless remains an ethical issue. This is also strongly suggested by the emotional reaction of humans working with animals, and having to decide over, and execute, their killing, as discussed in the third section of the paper.

The humans directly or indirectly involved in the killing of research animals seem to differentiate between different animal species, considering it more problematic to kill cats, dogs and non-human primates than rodents and rabbits. This differential attitude is not limited to the killing of animals but applies to animals in research in general, and it is consistent with the socio-zoological scale presented by Arluke and Sanders in which animals are ranked according to how much they are valued by humans, with companion animal species and non-human primates topping the list [64]. Consistent with this, there is also a greater investment in finding alternatives to killing animals in the case of dogs (usually re-homed into families) and chimpanzees (retired into sanctuaries).

But even for smaller species, such as rodents, the possibility of rehabilitation and rehoming, at least in some circumstances, may not be so far-fetched. It may in fact sometimes be easier to find a home for groups of these animals than for a single dog. From the perspective of the adopter, the small size, easy and affordable maintenance and short longevity means the commitment might be easier to take on. Also, from the animals' perspective, adaptation may be easier for a rodent who will be maintained with its social group than for a dog who needs to adapt from living primarily with dogs to be a single canine member of a human family. One interesting option for rodents is their use as classroom pets in schools [65]. This can be a useful resource for teaching values as responsibility and respect for animals as well as to dealing with loss [66]. When well managed and housed in large and species-appropriate habitats, the animals are provided better living conditions than in the animal facility. [67]. No matter whether the animals are adopted to families or to schools, it is of course important that the adopters consciously assume the responsibility to care for the animals as long as these live. Rehoming animals to initially friendly carers who lose interest in these animals in short time is neither in the animals' nor in the adopters' best interests.

Several issues, however, may prevent this practice from becoming mainstream. These include animal rehabilitation being labour demanding, costly and time consuming; the difficulty in ensuring that re-homed animals are housed, supervised and handled at least to the same standards found in animal facilities; and the onus of responsibility - as well as legal liability - in case animals are found to be mistreated. Animals may also manifest physical and behavioural abnormalities with a welfare impact, as a result of prolonged captivity or inadaptation to re-homing conditions [31, 68]. Therefore, rehabilitation of most laboratory animals may be unpractical or even unfeasible, thus often making euthanasia the ethically preferable option. However, although re-homing of research animals may presently sound outlandish to most, it nonetheless deserves more serious thought, in particular for the considerable number of surplus animals that do not need rehabilitation nor constitute a risk of disease transmission. After all, if animal research is usually portrayed to the public as only being carried out when no alternatives are available, should this not also hold for killing?

\section{Acknowledgements}

The authors wish to thank Dorte Bratbo Sørensen and Helena Rocklinsberg for constructive criticism of a previous version of this chapter.

\section{REFERENCES}

1. Crettaz von Roten, F., Public perceptions of animal experimentation across Europe. Public Understanding of Science, 2012.

2. Aldhous, P., A. Coghlan, and J. Copley, Animal experiments: let the people speak. . New Scientist., 1999 162(2187): p. 26-31.

3. European Commission, Seventh Report on the Statistics on the Number of Animals used for Experimental 
and other Scientific Purposes in the Member States of the European Union. 2013: Brussels.

4. Eurostat, Europe in Figures - Eurostat yearbook 2012. 2012, Publications Office of the European Union: Luxembourg. p. 119.

5. Eurostat (2009) EU cattle, pigs, sheep and goats: monthly slaughter statistics in 2008. Data in focus 15/2009.

6. Eurostat (2008) Poultry statistics in the European Union: flock numbers, hatcheries, trade and slaughterings. Data in focus $31 / 2008$.

7. Eurostat, Fishery Statistics, in Agriculture and fishery statistics - Main results 2010-11. 2011, Publications Office of the European Union: Luxembourg.

8. Cohen, C., The Case for the Use of Animals in Biomedical Research. New England Journal of Medicine, 1986. 315(14): p. 865-870.

9. Dell, R.B., S. Holleran, and R. Ramakrishnan, Sample size determination. ILAR J, 2002. 43(4): p. 207-13.

10. Bloomsmith, M.A., S.J. Schapiro, and E.A. Strobert, Preparing chimpanzees for laboratory research. ILAR J, 2006. 47(4): p. 316-25.

11. Davis, S.L., et al., Noninvasive pulmonary [18F]-2fluoro-deoxy-D-glucose positron emission tomography correlates with bactericidal activity of tuberculosis drug treatment. Antimicrob Agents Chemother, 2009. 53(11): p. 4879-84.

12. Davis, S.L., et al., Bacterial Thymidine Kinase as a Non-Invasive Imaging Reporter for $<$ italic $>$ Mycobacterium tuberculosis</italic $>$ in Live Animals. PLoS ONE, 2009. 4(7): p. e6297.

13. Andreu, N., P.T. Elkington, and S. Wiles, Molecular Imaging in TB: From the Bench to the Clinic, in Understanding Tuberculosis - Global Experiences and Innovative Approaches to the Diagnosis, P.-J. Cardona, Editor. 2012, InTech.

14. Morton, D.B., Humane endpoints in animal experimentation for biomedical research, in Humane endpoints in animals experiments for biomedical research C.F.M. Hendriksen and D.B. Morton, Editors. 1999, Royal Society of Medicine Press: London. p. 5-12.

15. Van Loo, P.L.P., et al., Preference for social contact versus environmental enrichment in male laboratory mice. Laboratory Animals, 2004. 38(2): p. 178-188.

16. Van Loo, P.L.P., L.F.M. Van Zutphen, and V. Baumans, Male management: coping with aggression problems in male laboratory mice. Laboratory Animals, 2003. 37(4): p. 300-313.

17. Beery, A.K. and I. Zucker, Sex bias in neuroscience and biomedical research. Neuroscience \&amp; Biobehavioral Reviews, 2011.35(3): p. 565-572.

18. Wald, C. and C. Wu, Of Mice and Women: The Bias in Animal Models. Science, 2010. 327(5973): p. 1571-1572.

19. Hawkins, P., et al., Husbandry refinements for rats, mice, dogs and non-human primates used in telemetry procedures. Seventh report of the BVAAWF/FRAME/RSPCA/UFAW Joint Working Group on Refinement, Part B. Lab Anim, 2004. 38(1): p. 1-10.
20. Weekley, L.B., P. Guittin, and G. Chamberland, The international symposium on regulatory testing and animal welfare: recommendations on best scientific practices for safety evaluation using nonrodent species. ILAR J, 2002. 43 Suppl: p. S118-22.

21. Stephens, M.L., et al., Possibilities for refinement and reduction: future improvements within regulatory testing. ILAR J, 2002. 43 Suppl: p. S74-9.

22. Broadhead, C.L., et al., Prospects for reducing and refining the use of dogs in the regulatory toxicity testing of pharmaceuticals. Hum Exp Toxicol, 2000. 19(8): p. 440-7.

23. Jennings, M., et al., Refinements in husbandry, care and common procedures for non-human primates: Ninth report of the BVAAWF/FRAME/RSPCA/UFAW Joint Working Group on Refinement. Lab Anim, 2009. 43 Suppl 1: p. 1-47.

24. Morton, D.B., et al., Refinements in telemetry procedures:seventh report of the BVA(AWF)/FRAME/RSPCA/UFAW joint working group on refinement, part A. Laboratory Animals, 2003. 37: p. 261-99.

25. Kramer, K. and L.B. Kinter, Evaluation and applications of radiotelemetry in small laboratory animals. Physiological Genomics, 2003. 13(3): p. 197-205.

26. Turner, P.V., et al., Refinements in the Care and Use of Animals in Toxicology StudiesRegulation, Validation, and Progress. Journal of the American Association for Laboratory Animal Science, 2003. 42(6): p. 8-15.

27. European Commission, Directive 2010/63/EU of the European Parliament and of the Council of 22 September 2010 on the protection of animals used for scientific purposes, E. Commission, Editor. 2010, Official Journal of the European Union: Brussels. p. 33-79.

28. Franco, N. and I. Olsson, Scientists and the 3Rs: attitudes to animal use in biomedical research and the effect of mandatory training in laboratory animal science. Laboratory animals, 2014. 48(1): p. 50-60.

29. Franco, N.H., M. Magalhães-Sant'Ana, and I.A.S. Olsson, Welfare and quantity of life, in Dilemmas in Animal Welfare, M. Appleby, D. Weary, and P. Sandøe, Editors. 2014, CABI: Oxfordshire. p. 46-66.

30. Wolfensohn, S., Euthanasia and Other Fates for Laboratory Animals, in The UFAW Handbook on the Care and Management of Laboratory and Other Research Animals, R. Hubrecht and J. Kirkwood, Editors. 2010, Wiley-Blackwell. p. 219-226.

31. Kerwin, A.M., Overcoming the Barriers to the Retirement of Old and New World Monkeys From Research Facilities. Journal of Applied Animal Welfare Science, 2006. 9(4): p. 337-347.

32. Waitt, C.D., M. Bushmitz, and P.E. Honess (2010) Designing Environments for Aged Primates. Laboratory Primate Newsletter 49

33. Carbone, L. (1997) Adoption of Research Animals. Animal Welfare Information Center Newsletter 7.

34. DiGangi, B.A., P.C. Crawford, and J.K. Levy, Outcome of Cats Adopted From a Biomedical Research Program. Journal of Applied Animal Welfare Science, 2006. 9(2): p. 143-163. 
35. Doehring, D. and M.H. Erhard, [Whereabouts of surplus and surviving laboratory animals]. ALTEX, 2005. 22(1): p. 7-11.

36. LASA, LASA Guidance on the Rehoming of Laboratory Dogs. A report based on a LASA working party and LASA meeting on rehoming laboratory animals, M. Jennings and B. Howard, Editors. 2004.

37. Taylor, K., The need for proactive rehoming policies in the EU. ALTEX Proceedings, 2015. 4(2): p. 228.

38. Kelch, T.G., Constitutional Protection for Animals, in Globalization and Animal Law: Comparative Law, International Law and International Trade. 2011, Kluwer Law International. p. 271-292.

39. Tierschutzgesetz. (TierSchG). (Animal Protection Act). December 9th 2010: BGBl. I S. 1934.

40. Franco, N.H., M. Correia-Neves, and I.A.S. Olsson, Animal welfare in studies on murine tuberculosis: assessing progress over a 12-year period and the need for further improvement. PloS one, 2012. 7(10): p. e47723.

41. Pereira, S. and M. Tettamanti, Ahimsa and alternatives - The concept of the 4th R. The CPCSEA in India. ALTEX, 2005. 22(1): p. 3-6.

42. Franco, N.H., Animal Experiments in Biomedical Research: A Historical Perspective. Animals, 2013. 3(1): p. 238-273.

43. Kant, I., Lectures on Ethics, J.B. Schneewind, Editor. 1997 [1780], Cambridge University Press.

44. Singer, P., Animal Liberation. 2002: Ecco.

45. Boralevi, L.C., Bentham and the Oppressed. 1984: W. de Gruyter.

46. McMahan, J., Eating animals the nice way. Daedalus, 2008. 137(1): p. 66-76.

47. Russell, W.M.S. and R.L. Burch, The principles of humane experimental technique 1959, London: Methuen \& Co. Ltd.

48. de Boo, M.J., et al., The interplay between replacement, reduction and refinement: considerations where the Three Rs interact. Animal Welfare, 2005. 14(4): p. 327332.

49. Hansen, A.K., et al., The need to refine the notion of reduction, in Humane endpoints in animal experiments for biomedical research, C. Hendriksen and D. Morton, Editors. 1999, RSM Press: London. p. 139-144.

50. Frey, R.G., Utilitarianism and Animals, in The Oxford Handbook of Animal Ethics, T.L. Beauchamp and R.G. Frey, Editors. 2011, OUP USA. p. 172-197.

51. McMahan, J., The Wrongness of Killing and the Badness of Death, in The Ethics of Killing: Killing at the Margins of Life. 2002, Oxford University Press. p. 189-202.

52. Rollin, B., Death, telos and euthanasia, in The end of animal life: a start for ethical debate. , F.L.B. Meijboom and E.N. Stassen, Editors. 2016, Wageningen Academic Publishers: Wageningen.

53. Rollin, B.E., Animal Rights as a Mainstream Phenomenon. Animals, 2011. 1(1): p. 102-115.
54. Iliff, S.A., An Additional "R": Remembering the Animals. ILAR Journal, 2002. 43(1): p. 38-47.

55. Calhoun, J.B., The Ecology and Sociology of the Norway Rat. 1963: U.S. Government Printing Office.

56. Altun, M., et al., Behavioral impairments of the aging rat. Physiology \&amp; Behavior, 2007. 92(5): p. 911-923.

57. Holmberg, T., A Feeling for the Animal: On Becoming an Experimentalist. Society and Animals, 2008. 16 p. 316-335

58. Bayne, K., Development of the human-research animal bond and its impact on animal well-being. ILAR J, 2002. 43(1): p. 4-9.

59. Chang, F.T. and L.H. Hart, Human-animal bonds in the laboratory: how animal behavior affects the perspectives of caregivers. ILAR J, 2002. 43(1): p. 10-8.

60. Cressey, D., Battle Scars. Nature, 2011. 470: p. 452453.

61. Arluke, A., Managing emotions in an animal shelter. 1994: Animals and human society. New York: Routledge.

62. Birke, L., A. Arluke, and M. Michael, The division of Emotional Labor, in The Sacrifice: How Scientific Experiments Transform Animals and People. 2007, Purdue University Press. p. 93-110.

63. Lynch, M.E., Sacrifice and the Transformation of the Animal Body into a Scientific Object: Laboratory Culture and Ritual Practice in the Neurosciences. Social Studies of Science, 1988. 18(2): p. 265-289.

64. Arluke, A. and C.R. Sanders, Regarding Animals. 1996: Temple University Press.

65. Baumans, V., et al., eds. Making Lives Easier for Animals in Research Labs. 2007, Animal Welfare Institute: Washington.

66. Huddart, S. and C. Naherniak, Animals in the Classroom, in Teaching Green: The Elementary Years : Handson Learning in Grades K-5, T. Grant and G. Littlejohn, Editors. 2005, New Society Publishers: Toronto. p. 101-107.

67. Fonseca, M.J., et al., Children's attitudes towards animals: evidence from the RODENTIA project. Journal of Biological Education, 2011. 45(3): p. 121-128.

68. Hubrecht, R., Comfortable quarters for laboratory dogs in research institutions, in Comfortable quarters for laboratory animals, V. Reinhardt and A. Reinhardt, Editors. 2002, Animal Welfare Institute. p. 56-64. 